\title{
One-parameter Family of Radon-Nikodym Theorems for States of a von Neumann Algebra
}

By

\author{
Huzihiro ARAKI
}

\begin{abstract}
It is shown that any normal state $\varphi$ of a von Neumann algebra $\mathfrak{M}$ with a cyclic and separating vector $\Psi$ satisfying $\varphi \leqq l \omega_{\Psi}$ for some $l>0$ has a representative vector $\Phi_{\alpha}$ in $V_{\Psi}^{\alpha}$ for each $\alpha \in[0,1 / 2]$ and $\Phi_{\alpha}=Q_{\alpha} \Psi$ for a $Q_{\alpha} \in \mathfrak{D}$ satisfying $\left\|Q_{\alpha}\right\| \leqq l^{1 / 2}$ when $\alpha \in[0,1 / 4]$.
\end{abstract}

\section{§1. Main Theorem}

Let $\mathfrak{M}$ be a von Neumann algebra on a Hilbert space $\mathfrak{H}$ with a unit cyclic and separating vector $\Psi$. Let $\Delta_{\Psi}$ be the modular operator for $\mathfrak{M}, \Psi$. Let $V_{\Psi}^{\alpha}$ denote the closure of $\Delta_{\Psi}^{\alpha} \mathfrak{M}^{+} \Psi$ where $\mathfrak{M}^{+}$denotes the positive operators in $\mathfrak{M}([2],[6])$.

Our main result is the following theorem:

Theorem 1. For any normal state $\varphi$ of $\mathfrak{M}$ such that $\varphi \leqq l \omega_{\Psi}$ for some $l>0$, there exists a vector $\Phi_{\alpha} \in V_{\Psi}^{\alpha}$ for every $\alpha \in[0,1 / 2]$ such that $\omega_{\Phi_{\alpha}}=\varphi$.

Combined with Theorem 3(8) of [2], Theorem 1 implies the following:

Theorem 2. For any normal state $\varphi$ of $\mathfrak{M}$ such that $\varphi \leqq l \omega_{\Psi}$, there exists a $Q_{\alpha} \in \mathfrak{M}$ for $\alpha \in[0,1 / 4]$ such that $\omega_{Q_{a} \Psi}=\varphi,\left\|Q_{\alpha}\right\| \leqq l^{1 / 2}$.

Operators $Q_{\alpha}$, such that $Q_{\alpha} \Psi \in V_{\Psi}^{\alpha}$, are characterized in Theorem $3(7)$ of [2] by the property that $\sigma_{t}^{\psi}\left(Q_{\alpha}\right)$ has an analytic continuation

Received July 13, 1973. 
$\sigma_{z}^{\psi}\left(Q_{\alpha}\right) \in \mathfrak{M}$ for $\operatorname{Im} z \in[0,2 \alpha]$ and $\sigma_{i \alpha}^{\psi}\left(Q_{\alpha}\right) \geqq 0$, where $\sigma_{t}^{\psi}$ denotes the modular automorphisms of $\mathfrak{M}$ relative to $\Psi$.

The special case $\alpha=0$ gives the non-commutative Radon-Nikodym derivative of Sakai [7]. The case $\alpha=1 / 4$ gives the Radon-Nikodym derivative satisfying the chain rule [2].

\section{§2. An Application of Carlson's Uniqueness Theorem}

Let $\mathrm{f}(z)$ be holomorphic for $\operatorname{Re} z \geqq 0$ and of exponential type: $|\mathrm{f}(z)|$ $\leqq M \mathrm{e}^{\tau|z|}$ for some $\tau>0$ and $M>0$. Let

$$
\mathrm{h}(\theta)=\varlimsup_{r \rightarrow \infty} r^{-1} \log \left|\widetilde{\mathrm{f}}\left(r e^{i \theta}\right)\right|, \quad|\theta| \leqq \pi / 2
$$

Carlson's theorem states that if $h(\pi / 2)+h(-\pi / 2)<2 \pi$, then $\mathrm{f}(n)=0$ for $n=0,1,2, \ldots$ implies $\mathrm{f}(z) \equiv 0 . \quad[5]$

If $\tilde{\mathrm{f}} \in \mathscr{D}(R)$ (the set of $C^{\infty}$-functions with a compact support) and

$$
\begin{aligned}
\mathrm{f}(t) & =(2 \pi)^{-1} \int \tilde{\mathrm{f}}(p) e^{-i p t} \mathrm{~d} p \\
Q(\mathrm{f}) & =\int \sigma_{s}^{\psi}(Q) \mathrm{f}(s) \mathrm{d} s
\end{aligned}
$$

then $\sigma_{t}^{\psi}(Q)$ has an analytic continuation to the $\mathfrak{M}$-valued entire function

$$
\sigma_{z}^{\psi}(Q(\mathrm{f}))=Q\left(\mathrm{f}_{z}\right), \quad \mathrm{f}_{z}(s)=\mathrm{f}(s-z)
$$

We have also

$$
\sigma_{z_{1}}^{\psi}\left(\sigma_{z_{2}}^{\psi}[Q(\mathrm{f})]\right)=\sigma_{z_{1}+z_{2}}^{\psi}(Q(\mathrm{f}))
$$

If $\operatorname{supp} \tilde{\mathrm{f}} \subset[-L, L]$, then

$$
|\mathrm{f}(t+i z)| \leqq M_{1} e^{L|\operatorname{Re} z|}\left(1+(t-\operatorname{Im} z)^{2}\right)^{-1}
$$

for some $M_{1}>0\left(M_{1}=2 \max \left(\|\tilde{\mathrm{f}}\|_{1},\left\|\tilde{\mathrm{f}}^{\prime \prime}\right\|_{1}\right)\right.$ for example). Hence

$$
\left\|\sigma_{i z}^{\psi}(Q(\mathrm{f}))\right\| \leqq M_{2} e^{L|\operatorname{Re} z|}\|Q\|
$$

for $M_{2}=M_{1} \pi$. 
Let $\tilde{\mathrm{f}} \in \mathscr{D}(R), 0 \leqq \tilde{\mathrm{f}}(p) \leqq 1$ and $\tilde{\mathrm{f}}(q)=1$ for $|q| \leqq 1$. Let

$$
\hat{\mathrm{f}}_{\lambda}(p)=\tilde{\mathrm{f}}(\lambda p), \quad \mathrm{f}_{\lambda}(t)=\lambda^{-1} \mathrm{f}(t / \lambda) .
$$

Then by the strong continuity of $\sigma_{t}^{\psi}(Q)$ in $t$, we have

$$
\lim _{\lambda \rightarrow+0} Q\left(\mathrm{f}_{\lambda}\right)=Q
$$

Lemma 1. Let $S$ be an invertible positive self-adjoint operator such that

$$
(Q(\mathrm{f}) x, S y)=\left(\sigma_{i \alpha}^{\psi}(Q(\mathrm{f})) S x, y\right)
$$

for all $\tilde{\mathrm{f}} \in \mathscr{D}(R), Q \in \mathfrak{M}$ and $x, y \in D_{S}$ where $D_{S}$ is any core of $S$ (namely $\left.\overline{S \mid D_{S}}=S\right)$. Then

$$
\left(Q(\mathrm{f}) x, e^{\bar{z} \log S} y\right)=\left(\sigma_{i \alpha z}^{\psi}(Q(\mathrm{f})) e^{z \log S} x, y\right)
$$

for all complex $z, \tilde{\mathrm{f}} \in \mathscr{D}(R), Q \in \mathfrak{M}, x$ in the domain of $e^{z \log S}$ and $y$ in the domain of $e^{\bar{z} \log S}$. For real $t$ and $Q \in \mathfrak{M}$,

$$
e^{i t \log S} Q e^{-i t \log S}=\sigma_{-\alpha t}^{\psi}(Q)
$$

Proof. By a limiting procedure, (2.8) holds for all $x$ and $y$ in the domain of $S$. Let $D_{\mathrm{a}}$ be the set of all vectors which have compact supports relative to the spectrum of $\log S$. For any $x$ and $y$ in $D_{a}$, $e^{z \log S} x$ and $e^{z \log S} y$ are vector-valued entire functions of $z$ and

$$
\begin{gathered}
\left\|e^{z \log S} x\right\| \leqq M_{x} e^{a|\operatorname{Re} z|}, \\
\left\|e^{\bar{z} \log S} y\right\| \leqq M_{y} e^{b|\operatorname{Re} z|},
\end{gathered}
$$

for some $a>0, b>0, M_{x}>0$ and $M_{y}>0$. From (2.11), (2.12) and (2.6), it follows that both sides of (2.9) are entire functions of exponential type with $\mathrm{h}(\pi / 2)=\mathrm{h}(-\pi / 2)=0$. If $x, y \in D_{\mathrm{a}}$, then $e^{m \log S} x=S^{m} \quad x \in D_{\mathrm{a}}$ and $e^{m \log S} y=S^{m} y \in D_{\mathrm{a}}$. Hence, by repeated use of (2.8), we have (2.9) for $z=0,1,2, \ldots$ By Carlson's theorem, (2.9) holds for all $z$ and $x, y \in D_{\mathrm{a}}$. Since $D_{\mathrm{a}}$ is a core of $e^{\lambda \log S}$ for any real $\lambda$, and since $e^{i \lambda \log S}$ is bounded for real $\lambda$, (2.9) holds as stated in the Lemma. 
By a limiting procedure like (2.7), we obtain (2.10) from (2.9). Q.E.D.

Lemma 2. If a self-adjoint operator $S \geqq 0$ satisfies (2.8) for all $\tilde{\mathrm{f}} \in \mathscr{D}(R), Q \in \mathfrak{M}$ and $x, y \in D_{S}$, then

$$
\left(Q(\mathrm{f}) x, S^{1 / 2} y\right)=\left(\sigma_{i \alpha / 2}^{\psi}(Q(\mathrm{f})) S^{1 / 2} x, y\right)
$$

for all $\tilde{\mathrm{f}} \in \mathscr{D}(R), Q \in \mathfrak{M}, x$ and $y$ in the domain of $S^{1 / 2}$.

Proof. Let $E$ be the projection onto the null space of $S$. By setting $x=(1-E) x^{\prime}$ in $(2.8)$ for arbitrary $x^{\prime}$, we obtain

$$
\left(Q(\mathrm{f})(1-E) x^{\prime}, S y\right)=0 .
$$

Hence $E Q(\mathrm{f})(1-E)=0$. Replacing $Q$ by $Q^{*}$, $\mathrm{f}$ by $\mathrm{f}^{*}$ and taking the adjoint, we also have $(1-E) Q(\mathrm{f}) E=0$. Hence $E Q(\mathrm{f})=E Q(\mathrm{f}) E=Q(\mathrm{f}) E$. Since the set of $Q(f)$ is dense in $\mathfrak{M}$ by (2.7), we have $E \in \mathfrak{M}^{\prime}$.

Now the proof of Lemma 1 holds for $x, y \in E \mathfrak{H}$, and hence we have (2.9) whenever $\tilde{\mathrm{f}} \in \mathscr{D}(R), Q \in \mathfrak{M}, x \in E \mathfrak{S}, y \in E \mathfrak{H}, x$ is in the domain of $e^{z \log S} E$ and $y$ in the domain of $e^{\bar{z} \log S} E$. Setting $z=1 / 2$, and using $[E, Q(\mathrm{f})]=\left[E, \sigma_{i \alpha z}^{\psi}(Q(\mathrm{f}))\right]=0$, we have (2.13).

Q.E.D.

\section{$\S 3$ Basic Lemmas}

For any closable linear operator $A$ with a dense domain, let $|A|=$ $\left(A^{*} \bar{A}\right)^{1 / 2}$ and $\mathrm{u}(A)=\left(|A|^{-1} A^{*}\right)^{*}$, where the bar denotes the closure. The operator $\mathrm{u}(A)$ is a partial isometry, whose kernel is the kernel of $|A|$, and $\bar{A}=\mathrm{u}(A)|A|$ is the polar decomposition of $A$.

Lemma 3 Let $A_{1}$ and $B_{1}$ be closed linear operators affiliated with $\mathfrak{M}, A_{2}$ and $B_{2}$ be closed linear operators affiliated with $\mathfrak{M}^{\prime}$, and $\alpha$ be a real number. Assume that either one of the following conditions holds:

(1) $\alpha \in[0,1 / 2], \Psi$ is in the domains of $A_{j}, A_{j}^{*}, B_{j}$ and $B_{j}^{*}, j=$ $1,2$.

(2) $\Psi$ is in the domains of $A_{1}, \Delta_{\Psi}^{\alpha} A_{1}^{*}, \Delta_{\Psi}^{\alpha} B_{1}, B_{1}^{*}, A_{2}, \Delta_{\Psi}^{-\alpha} A_{2}^{*}, \Delta_{\Psi}^{-\alpha} B_{2}$ and $B_{2}^{*}$. 
Then $A_{1} \Delta_{\Psi}^{\alpha}, \Delta_{\Psi}^{\alpha} B_{1}, A_{2} \Delta_{\Psi}^{-\alpha}, \Delta_{\Psi}^{-\alpha} B_{2}$ are closable linear operators with dense domains.

Proof. Let $\mathfrak{H}_{\Psi_{1}}$ and $\mathfrak{A}_{\Psi_{2}}$ be the *-algebras of all operators $Q_{1} \in \mathfrak{M}$ and $Q_{2} \in \mathfrak{M}^{\prime}$, respectively, such that $\bar{\sigma}_{t}^{\psi}\left(Q_{j}\right) \equiv \Delta_{\Psi}^{i t} Q \Delta_{\Psi}^{-i t}$ have analytic continuations to entire functions $\bar{\sigma}_{z}^{\psi}\left(Q_{j}\right), j=1,2$. [2].

If $Q_{2} \in \mathfrak{A}_{\Psi_{2}}$, then $Q_{2} \Psi$ is in the domains of $A_{1} \Delta_{\Psi}^{\alpha},\left(A_{1} \Delta_{\Psi}^{\alpha}\right)^{*}, \Delta_{\Psi}^{\alpha} B_{1}$ and $\left(\triangle_{\Psi}^{\alpha} B_{1}\right)^{*}$ :

$$
\begin{aligned}
& \left(A_{1} \Delta_{\Psi}^{\alpha}\right) Q_{2} \Psi=\bar{\sigma}_{-i \alpha}^{\psi}\left(Q_{2}\right) A_{1} \Psi, \\
& \left(A_{1} \Delta_{\Psi}^{\alpha}\right)^{*} Q_{2} \Psi=\bar{\sigma}_{-i \alpha}^{\psi}\left(Q_{2}\right) \Delta_{\Psi}^{\alpha} A_{1}^{*} \Psi, \\
& \left(\Delta_{\Psi}^{\alpha} B_{1}\right) Q_{2} \Psi=\bar{\sigma}_{-i \alpha}^{\psi}\left(Q_{2}\right) \Delta_{\Psi}^{\alpha} B_{1} \Psi, \\
& \left(\Delta_{\Psi}^{\alpha} B_{1}\right)^{*} Q_{2} \Psi=\bar{\sigma}_{-i \alpha}^{\psi}\left(Q_{2}\right) B_{1}^{*} \Psi,
\end{aligned}
$$

where $A_{1}^{*} \Psi$ and $B_{1} \Psi$ are in the domain of $\Delta_{\Psi}^{\alpha}$ for $\alpha \in[0,1 / 2]$ due to

$$
\Delta_{\Psi}^{1 / 2} A \Psi=J_{\Psi} A^{* \Psi}
$$

for any $A$ affiliated with $\mathfrak{M}$ and for $\Psi$ in the domains of $A$ and $A^{*}$, as can be easily proved by a polar decomposition of $A$ and spectral resolution of $|A|$. Since $\mathfrak{H}_{\Psi_{2}} \Psi$ is dense, $A_{1} \Delta_{\Psi}^{\alpha}$ and $\Delta_{\Psi}^{\alpha} B_{1}$ are closable linear operators with dense domains.

Similarly, $\mathfrak{A}_{\Psi_{1}} \Psi$ is in the domains of $A_{2} \Delta_{\Psi^{-\alpha}}^{-\alpha}\left(A_{2} \Delta_{\Psi}^{-\alpha}\right)^{*}, \Delta_{\Psi}^{-\alpha} B_{2},\left(\Delta_{\Psi}^{-\alpha}\right.$ $\left.B_{2}\right)^{*}$ and hence $A_{2} \Delta_{\Psi}^{-\alpha}$ and $\Delta_{\Psi}^{-\alpha} B_{2}$ are closable linear operators with dense domains.

Q.E.D.

Lemma 4. Let $A_{1}$ and $B_{1}$ be closed linear operators affiliated with $\mathfrak{M}$ and $A_{2}$ and $B_{2}$ be closed linear operators affiliated with $\mathfrak{M}^{\prime}$, such that $A_{1} \Delta_{\Psi}^{\alpha}, \Delta_{\Psi}^{\alpha} B_{1}, A_{2} \Delta_{\Psi}^{-\alpha}, \Delta_{\Psi}^{-\alpha} B_{2}$ are closable linear operators with dense domains. Then

$$
\begin{aligned}
& \mathrm{u}\left(A_{1} \Delta_{\Psi}^{\alpha}\right) \in \mathfrak{M}, \quad \mathrm{u}\left(\Delta_{\Psi}^{\alpha} B_{1}\right) \in \mathfrak{M}, \\
& \mathrm{u}\left(A_{2} \Delta_{\Psi}^{-\alpha}\right) \in \mathfrak{M}^{\prime}, \quad \mathrm{u}\left(\Delta_{\Psi}^{-\alpha} B_{2}\right) \in \mathfrak{M}^{\prime}
\end{aligned}
$$

Here $\alpha$ is real. 
Proof. For $T=A_{2} \Delta_{\Psi^{\alpha}}^{-\alpha}$ or $\Delta_{\Psi^{\alpha}} B_{2}$, we have

$$
Q(\mathrm{f})^{*} \bar{T} y=\bar{T} \sigma_{-i \alpha}^{\psi}\left(Q(\mathrm{f})^{*}\right) y
$$

for $y$ in the domain of $T$ and hence for $y$ in the domain of $\bar{T}$. Since $\sigma_{-i \alpha}^{\psi}\left(Q(\mathrm{f})^{*}\right)^{*}=\sigma_{i \alpha}^{\psi}(Q(\mathrm{f}))$, we have

$$
(Q(\mathrm{f}) x, \bar{T} y)=\left(\sigma_{i \alpha}^{\psi}(Q(\mathrm{f})) T^{*} x, y\right)
$$

for all $x$ in the domain of $T^{*}$ and $y$ in the domain of $\bar{T}$. We also have

$$
\begin{aligned}
\left(Q(\mathrm{f}) y, T^{*} x\right) & =\left(Q(\mathrm{f})^{*} T^{*} x, y\right)^{*}=\left(\sigma_{-i \alpha}^{\psi}\left(Q(\mathrm{f})^{*}\right) x, \bar{T} y\right)^{*} \\
& =\left(\sigma_{i \alpha}^{\psi \prime}(Q(\mathrm{f})) \bar{T} y, x\right)
\end{aligned}
$$

for $x$ in the domain of $T^{*}$ and $y$ in the domain of $\bar{T}$. Hence the positive self-adjoint operator $S=T^{*} \bar{T}$ satisfies

$$
(Q(\mathrm{f}) x, S y)=\left(\sigma_{2 i \alpha}^{\psi}(Q(\mathrm{f})) S x, y\right)
$$

for all $x$ and $y$ in the domain of $S$. (See (2.5).) By Lemma 2, $|T|=$ $S^{1 / 2}$ satisfies

$$
(Q(\mathrm{f}) x,|T| y)=\left(\sigma_{i \alpha}^{\psi}(Q(\mathrm{f}))|T| x, y\right)
$$

for all $x$ and $y$ in the domain of $|T|$. From (3.8) and (3.10), we have

$$
\begin{aligned}
(Q(\mathrm{f}) x, \mathrm{u}(T)|T| y) & =\left(\sigma_{i \alpha}^{\psi}(Q(\mathrm{f}))|T||T|^{-1} T^{*} x, y\right) \\
& =\left(Q(\mathrm{f}) \mathrm{u}(T)^{*} x,|T| y\right)
\end{aligned}
$$

for $x$ in the domain of $T^{*}$ and $y$ in the domain of $|T|$.

Since $\mathrm{u}(T)^{*} \mathrm{u}(T)$ is the projection onto the closure of the range of $|T|$, we have

$$
Q(\mathrm{f}) * \mathrm{u}(T)=\mathrm{u}(T) Q(\mathrm{f}) * \mathrm{u}(T) * \mathrm{u}(T)
$$

$1-\mathrm{u}(T)^{*} \mathrm{u}(T)$ is the projection onto the kernel of $T$ and hence onto the kernel of $S=T^{*} T$. By the proof of Lemma 2, (3.9) implies that $\left[Q(\mathrm{f}), \mathrm{u}(T)^{*} \mathrm{u}(T)\right]=0$. Hence (3.11) implies

$$
Q(\mathrm{f})^{*} \mathrm{u}(T)=\mathrm{u}(T) \mathrm{u}(T)^{*} \mathrm{u}(T) Q(\mathrm{f})=\mathrm{u}(T) Q(\mathrm{f}) .
$$


Hence $\mathrm{u}(T) \in \mathfrak{M}^{\prime}$.

A similar proof holds for $A_{1} \Delta_{\Psi}^{\alpha}$ and $\Delta_{\Psi}^{\alpha} B_{1}$, where $\mathfrak{M}$ is replaced by $\mathfrak{M}^{\prime}$.

Q.E.D.

Lemma 5. The vectors

$$
Q(\mathrm{f}) \sigma_{-2 i \alpha}^{\psi}\left(Q(\mathrm{f})^{*}\right) \Psi=Q(\mathrm{f}) \Delta_{\Psi}^{2 \alpha} Q(\mathrm{f}) * \Psi
$$

for $Q \in \mathfrak{M}$ and $\tilde{\mathrm{f}} \in \mathscr{D}(R)$ are in $V_{\Psi}^{\alpha}$ and dense in $V_{\Psi}^{\alpha}$ for $\alpha \in[0,1 / 2]$. The vectors

$$
Q^{\prime}(\mathrm{f}) \bar{\sigma}_{i-2 i \alpha}^{\prime}\left(Q^{\prime}(\mathrm{f})^{*}\right)=Q^{\prime}(\mathrm{f}) \Delta_{\Psi}^{2 \alpha-1} Q^{\prime}(\mathrm{f})^{*} \Psi
$$

for $Q^{\prime} \in \mathfrak{M}^{\prime}$ and $\tilde{\mathrm{f}} \in \mathscr{D}(R)$ are in $V_{\Psi}^{\alpha}$ and dense in $V_{\Psi}^{\alpha}$ for $\alpha \in[0,1 / 2]$.

Proof. Since $q=Q(\mathrm{f}) \sigma_{-2 i \alpha}^{\psi}\left(Q(\mathrm{f})^{*}\right)$ has an analytic continuation

$$
\sigma_{z}^{\psi}(q)=\sigma_{z}^{\psi}[Q(\mathrm{f})]\left(\sigma_{\bar{z}+2 i \alpha}^{\psi}[Q(\mathrm{f})]\right)^{*}
$$

which is obviously positive for $z=i \alpha, q \Psi$ is in $V_{\Psi}^{\alpha}$ by Theorem 3 (7) of $[2]$.

By definition, $\Delta_{\Psi}^{\alpha} Q^{2} \Psi, Q \in \mathfrak{M}^{+}$is dense in $V_{\Psi}^{\alpha}$. If $Q\left(\mathrm{f}_{\lambda}\right)^{*}=Q\left(\mathrm{f}_{\lambda}\right)$ are uniformly bounded and $Q\left(\mathrm{f}_{\lambda}\right) \rightarrow Q$ strongly, then $Q\left(\mathrm{f}_{\lambda}\right)^{2} \rightarrow Q^{2}$ strongly. Since $d(\alpha)=\left\|\Delta_{\Psi}^{\alpha}\left(Q\left(\mathrm{f}_{\lambda}\right)^{2}-Q^{2}\right) \Psi\right\|^{2}$ is convex in $\alpha$ and $d(0)=d(1 / 2) \rightarrow 0$, we have $\Delta_{\Psi}^{\alpha} Q\left(\mathrm{f}_{\lambda}\right)^{2} \Psi \rightarrow \Delta_{\Psi}^{\alpha} Q^{2} \Psi$. Hence the vectors

$$
\Delta_{\Psi}^{\alpha} Q(\mathrm{~g})^{2} \Psi=Q(\mathrm{f}) \sigma_{-2 i \alpha}^{\psi}\left(Q(\mathrm{f})^{*}\right) \Psi
$$

for $Q=Q^{*} \in \mathfrak{M}, \tilde{\mathrm{g}} \in \mathscr{D}(R)$ and $\mathrm{g}^{*}=\mathrm{g}$ are dense in $V_{\Psi}^{\alpha}$ where $\mathrm{f}(t)=\mathrm{g}(t+$ $i \alpha), \sigma_{-i \alpha}^{\psi}(Q(\mathrm{~g}))=Q(\mathrm{f})$. This completes the proof of the first half.

The second half is obtained from the first half by

$$
J_{\Psi} Q(\mathrm{f}) \sigma_{-2 i \beta}^{\psi}\left(Q(\mathrm{f})^{*}\right) \Psi=Q^{\prime}\left(\mathrm{f}^{*}\right) \bar{\sigma}_{2 i \beta}^{\psi}\left(Q^{\prime}\left(\mathrm{f}^{*}\right)^{*}\right) \Psi
$$

for $\quad Q^{\prime}=J_{\Psi} Q J_{\Psi} \in \mathfrak{M i}^{\prime}\left(Q=J_{\Psi} Q^{\prime} J_{\Psi}\right)$ and $\beta=(1 / 2)-\alpha$, due to $J_{\Psi} V_{\Psi}^{\beta}=V_{\Psi}^{\alpha}$ (Theorem 3(4) of [2]).

Q.E.D.

Lemma 6. Let $A_{1}, A_{2}, B_{1}, B_{2}$ be as in Lemma 3 and $\alpha \in[0,1]$. Then

$$
\left|A_{1} \Delta_{\Psi}^{\alpha}\right| \Psi \in V_{\Psi}^{\alpha / 2}, \quad\left|\Delta_{\Psi}^{\alpha} B_{1}\right| \Psi \in V_{\Psi}^{\alpha / 2}
$$




$$
\left|A_{2} \Delta_{\Psi}^{-\alpha}\right| \Psi \in V_{\Psi}^{(1-\alpha) / 2}, \quad\left|\Delta_{\Psi}^{-\alpha} B_{2}\right| \Psi \in V_{\Psi}^{(1-\alpha) / 2}
$$

Proof. Since $\mathfrak{A}_{\Psi_{1}} \Psi$ is in the domain of $T$ for $T=A_{2} \Delta_{\Psi}^{-\alpha}$ and for $T=\Delta_{\Psi}^{-\alpha} B_{2}$, it is also in the domain of $|T|$. For $Q \in \mathfrak{M}$ and $\tilde{\mathrm{f}} \in \mathscr{D}(R)$, we have

$$
\begin{aligned}
& \left(Q(\mathrm{f}) \sigma_{-i \alpha}^{\psi}\left(Q(\mathrm{f})^{*}\right) \Psi,|T| \Psi\right)=\left(\sigma_{i \alpha}^{\psi}(Q(\mathrm{f}))|T| \sigma_{-i \alpha}^{\psi}\left(Q(\mathrm{f})^{*}\right) \Psi, \Psi\right) \\
& \quad=\left(|T| \sigma_{-i \alpha}^{\psi}\left(Q(\mathrm{f})^{*}\right) \Psi, \sigma_{-i \alpha}^{\psi}\left(Q(\mathrm{f})^{*}\right) \Psi\right) \geqq 0
\end{aligned}
$$

by (3.10). Since $Q(\mathrm{f}) \sigma_{-i \alpha}^{\psi}\left(Q(\mathrm{f})^{*}\right) \Psi$ are dense in $V_{\Psi}^{\alpha / 2}$, we have $|T| \Psi \in$ $V_{\Psi}^{(1-\alpha) / 2}$ due to Theorem 3(5) of [2].

Similarly we have

$$
\begin{aligned}
& \left(Q^{\prime}(\mathrm{f}) \bar{\sigma}_{i \alpha}^{\psi}\left(Q^{\prime}(\mathrm{f})^{*}\right) \Psi,\left|T^{\prime}\right| \Psi\right) \\
& \quad=\left(\left|T^{\prime}\right| \bar{\sigma}_{i \alpha}^{\psi}\left(Q^{\prime}(\mathrm{f})^{*}\right) \Psi, \bar{\sigma}_{i \alpha}^{\psi}\left(Q^{\prime}(\mathrm{f})^{*}\right) \Psi \geqq 0\right.
\end{aligned}
$$

for $T^{\prime}=A_{1} \Delta_{\Psi}^{\alpha}$ and for $T^{\prime}=\Delta_{\Psi}^{\alpha} B_{1}$. Hence $\left|T^{\prime}\right| \Psi \in\left(V_{\Psi}^{(1-\alpha) / 2}\right)^{\prime}=V_{\Psi}^{\alpha / 2}$.

Q.E.D.

\section{§. Proof of Theorem $\mathbb{1}$}

A vector $\Phi$ is called a representative of a state $\varphi$ if the vector state $\omega_{\Phi}$ is $\varphi$.

Lemma 7. If normal state $\varphi$ has a representative vector in $V_{\Psi^{1 / 2}}$, then it has a representative vector $\Phi_{\alpha}$ in $V_{\Psi}^{\alpha}$ for each $\alpha \in[1 / 4,1 / 2]$.

Proof. Let $\Phi \in V_{\Psi}^{1 / 2}$ and $\omega_{\Phi}=\varphi$. There exists a self-adjoint operator $A_{2} \geqq 0$ affiliated with $\mathfrak{M}^{\prime}$ such that $\Psi$ is in the domain of $A_{2}\left(=A_{2}^{*}\right)$ and $\Phi=A_{2} \Psi$. [8]. By Lemma $3, \mathrm{~A}_{2} \Delta_{\Psi}^{\beta}$ is a closable linear operator with a dense domain for $0 \leqq \beta \leqq 1 / 2$. Let

$$
\Phi_{\alpha}=\left|A_{2} \Delta_{\Psi}^{-\beta}\right| \Psi, \quad \alpha=(1-\beta) / 2 \in[1 / 4,1 / 2]
$$

By Lemma 6, $\Phi_{\alpha} \in V_{\Psi}^{\alpha} . \quad$ By Lemma $4, \quad u \equiv \mathrm{u}\left(A_{2} \Delta_{\Psi}^{-\beta}\right) \in \mathfrak{M}^{\prime} . \quad$ Furthermore, $u^{*} u\left|A_{2} \Delta_{\Psi}^{-\beta}\right|=\left|A_{2} \Delta_{\Psi}^{-\beta}\right|$ and

$$
u \Phi_{\alpha}=u\left|A_{2} \Delta_{\Psi}^{-\beta}\right| \Psi=A_{2} \Delta_{\Psi}^{-\beta} \Psi=A_{2} \Psi=\Phi .
$$


Hence, as states of $\mathfrak{M}$, we have the following equalities:

$$
\varphi=\omega_{\Phi}=\omega_{\Phi_{\alpha}} .
$$

Q.E.D.

Lemma 8. If $Q^{\prime} \in \mathfrak{M}^{\prime}$ and $Q^{\prime} \Psi \in V_{\Psi^{1 / 4}}^{1 / 4}$, then $\left(Q^{\prime}\right)^{*} \Psi$ is in the domain of $\Delta_{\Psi}^{-1}$.

Proof. By Theorem 4(2) of [2],

$$
Q^{\prime} \Psi=J_{\Psi} Q^{\prime} \Psi=\Delta_{\Psi}^{-1 / 2}\left(Q^{\prime}\right)^{*} \Psi
$$

Since $Q^{\prime} \Psi$ is in the domain of $\Delta_{\Psi^{1 / 2}}^{-1}$ for any $Q^{\prime} \in \mathfrak{M}^{\prime},\left(Q^{\prime}\right)^{*} \Psi$ must be in the domain of $\Delta \bar{\psi}^{1}$.

Q.E.D.

Proof of Theorem 1: It is well-known that $\varphi \leqq l \omega_{\Psi}$ implies the existence of $A_{2} \in\left(\mathfrak{M}^{\prime}\right)^{+}$such that $\Phi=A_{2} \Psi \quad\left(\in V_{\Psi}^{1 / 2}\right)$ is a representative of $\varphi$. By Lemma 7, $\varphi$ has a representative $\Phi_{1 / 4}$ in $V_{\Psi}^{1 / 4}$. (This is also obtained in Theorem 6 of [2].) By Theorem 3(8) of [2], there exists $Q \in \mathfrak{M}$ such that $\Phi_{1 / 4}=Q \Psi$. By Theorem $4(2)$ of [2], $\Phi_{1 / 4}=J_{\Psi} \Phi_{1 / 4}$ $=\left(J_{\Psi} Q J_{\Psi}\right) \Psi$. Set $Q^{\prime}=J_{\Psi} Q J_{\Psi} \in \mathfrak{M}^{\prime}$. By Lemma $8\left(Q^{\prime}\right)^{*} \Psi$ is in the domain of $\Delta_{\Psi}^{-1}$ and hence in the domain of $\Delta_{\Psi}^{-\beta}$ for any $\beta \in[0,1]$. By Lemma 3, $Q^{\prime} \Delta_{\Psi}^{-\beta}$ is a closable linear operator with a dense domain. Let

$$
\Phi_{\alpha}=\left|Q^{\prime} \Delta_{\Psi}^{-\beta}\right| \Psi, \quad \alpha=(1-\beta) / 2 \in[0,1 / 2] .
$$

By the same argument as the proof of Lemma $7, \Phi_{\alpha}$ is a representative vector of the state $\varphi$ in $V_{\Psi}^{\alpha}$.

Q.E.D.

Remark. If $\varphi \leqq l \omega_{\Psi}$, then there exists $A_{2} \in\left(\mathfrak{M}^{\prime}\right)^{+}$such that $\left\|A_{2}\right\| \leqq$ $l^{1 / 2}$ and $\omega_{\Phi}=\varphi$ for $\Phi=A_{2} \Psi$. For any $\alpha \in[0,1 / 2], \omega_{\Phi}=\omega_{\Phi_{\alpha}}$ implies the existence of a partial isometry $v_{\alpha} \in \mathfrak{M}^{\prime}$ such that $\Phi_{\alpha}=v_{\alpha} \Phi=Q_{\alpha} \Psi$, $Q_{\alpha}=v_{\alpha} A_{2}$. Then $Q_{\alpha} \in \mathfrak{M}^{\prime}$ and $\left\|Q_{\alpha}\right\| \leqq l^{1 / 2}$.

\section{§5. Additional Remarks}

The following Lemma is a variation of Lemma 6, which will be used in [4]. 
Lemma 9. Let $h \in \mathfrak{M l}^{+}$. Then

$$
\left|h^{1 / n} \Delta_{\Psi}^{1 /(2 n)}\right| n \Psi \in V_{\Psi^{\prime}}^{1 / 4}
$$

Proof. By setting $T=h^{1 / n} \Delta_{\Psi}^{1 /(2 n)}$ and replacing $Q \in \mathfrak{M}$ by $Q^{\prime} \in \mathfrak{M}^{\prime}$ in the proof of Lemma 4, we obtain

$$
\left(Q^{\prime}(\mathrm{f}) x,|T| y\right)=\left(\sigma_{-i /(2 n)}\left(Q^{\prime}(\mathrm{f})\right)|T| x, y\right)
$$

for all $x$ and $y$ in the domain of $|T|$. (cf. (3.10).) By repeated use, we have

$$
\left(Q^{\prime}(\mathrm{f}) x,|T|^{n} y\right)=\left(\sigma_{-i / 2}^{\psi}\left(Q^{\prime}(\mathrm{f})\right)|T|^{n} x, y\right)
$$

for all $x$ and $y$ in the domain of $|T|^{n}$.

By replacing $|T|$ by $|T|^{n}, Q \in \mathfrak{M}$ by $Q^{\prime} \in \mathfrak{M}^{\prime}, \sigma_{-i \alpha}^{\psi}$ by $\quad \bar{\sigma}_{i-i \alpha}^{\psi}$ and setting $\alpha=1 / 2$ in the proof of Lemma 6 , we obtain

$$
|T|^{n} \Psi \in\left(V_{\Psi}^{1 / 4}\right)^{\prime}=V_{\Psi}^{1 / 4} .
$$

Q.E.D.

\section{References}

[1] Araki, H., Bures distance function and a generalization of Sakai's non-commutative Radon-Nikodym theorem, Publ. RIMS Kyoto Univ. 8, 335-362 (1972).

[2] Araki, H., Some properties of modular conjugation operator of von Neumann algebras and a non-commutative Radon-Nikodym theorem with a chain rule, Pacific J. Math. 50, 309-354 (1974).

[3] Araki, H., Relative Hamiltonian for faithful normal states of a von Neumann algebra, Publ. RIMS Kyoto Univ. 9, 165-209(1973).

[4] Araki, H., Golden-Thompson and Peierls-Bogolubov inequalities for a general von Neumann algebra, Comm. Math. Phys. 34, 167-178 (1973).

[5] Boas, R. P., Entire functions, (Academic Press, New York, 1954).

[6] Connes, A., Groupe modulaire d'une algèbre de von Neumann, C. R. Acad. Sci. Paris Série A, 274, 1923-1926 (1972).

[7] Sakai, S., A Radon-Nikodym theorem in $W^{*}$-algebras, Bull. Amer. Math. Soc. 71, 149-151 (1965).

[8] Takesaki, M., Tomita's theory of modular Hilbert algebras and its applications, (Springer Verlag, Berlin-Heidelberg-New York, 1970). 